\title{
Left Ventricle Tracking Using Overlap Priors
}

\author{
Ismail Ben Ayed ${ }^{1}$, Yingli Lu${ }^{1}$, Shuo Li ${ }^{1}$, and Ian Ross ${ }^{2}$ \\ GE Healthcare, London, ON, Canada \\ London Health Sciences Centre, London, ON, Canada
}

\begin{abstract}
This study investigates overlap priors for tracking the Left Ventricle (LV) endo- and epicardium boundaries in cardiac Magnetic Resonance (MR) sequences. It consists of evolving two curves following the Euler-Lagrange minimization of two functionals each containing an original overlap prior constraint. The latter measures the conformity of the overlap between the nonparametric (kernel-based) intensity distributions within the three target regions-LV cavity, myocardium and background-to a prior learned from a given segmentation of the first frame. The Bhattacharyya coefficient is used as an overlap measure. Different from existing intensity-driven constraints, the overlap priors do not assume implicitly that the overlap between the distributions within different regions has to be minimal. Although neither shape priors nor curve coupling were used, quantitative evaluation showed that the results correlate well with independent manual segmentations and the method compares favorably with other recent methods. The overlap priors lead to a LV tracking which is more versatile than existing methods because the solution is not bounded to the shape/intensity characteristics of a training set. We also demonstrate experimentally that the used overlap measures are approximately constant over a cardiac sequence.
\end{abstract}

\section{Introduction}

Tracking the Left Ventricle (LV) endo- and epicardium boundaries in cardiac Magnetic Resonance (MR) sequences plays an essential role in diagnosing cardiovascular diseases. It consists of segmenting each frame into three regions: LV cavity, myocardium and background. Manual tracing is time-consuming. Therefore, an automatic tracking is desired. Although several techniques have addressed this task [2]-77, 9]-[11, accurate LV tracking is still acknowledged as a difficult problem because of the overlap between the intensity distributions within the cardiac regions (cf. the typical example in Fig. 1), the lack of edge information and the intensity/shape variability from one patient to another [10].

As discussed by Freedman et al. in [15], most of existing methods in medical image segmentation compute a pixelwise correspondence between the current image (or frame) and model distributions of shape and appearance (or intensity). Model distributions are generally learned from a training set and embedded in the segmentation via two standard frameworks: variational level-sets [1] (such as [2]-7]) and active appearance/shape models [8] (such as [9]-11]). 
In the level-set framework [2]-[7], the problem is commonly stated as the minimization of a functional containing two constraints: a shape prior constraint and an intensity-driven constraint based on the Maximum Likelihood (ML) principle. The latter maximizes the conditional probability of pixel intensity given the assumed model distribution within each region. Unfortunately, a ML intensitydriven constraint is sensitive to inaccuracies in estimating model distributions 12. More importantly, it can not incorporate information about the overlap between the intensity distributions within different regions. Based on the evaluation of a pixelwise correspondence between the image and the models, ML intensity-driven constraints assume implicitly that the overlap between the distributions within different regions has to be minimal. The pixelwise information is misleading in the case of the LV due to the "significant" (cf. the typical example in Fig. (1) overlap between the distributions within the cardiac regions. Consequently, the use of geometric priors (such as shape) in conjunction with ML intensity-driven constraints was inevitable to obtain satisfying results 5, 6. Similar to variational level-set approaches, active appearance/shape models compute a pixelwise correspondence between the image and the models [15. As we will show in the experiments (section 3 ), embedding global information about the overlap between the intensity distributions within the segmentation regions is important. In the current study, we devise overlap priors for LV tracking.

The current study is most related to recent variational segmentation/tracking methods 12-14 using similarity/dissimilarity measures between intensity distributions. Using the Bhattacharyya coefficient as an overlap measure, we propose to track the endo- and epicardium boundaries in a cardiac MR sequence by evolving two active curves following the Euler-Lagrange minimization of two functionals each containing an original overlap prior. The latter measures the conformity of the overlap between the nonparametric (kernel-based) intensity distributions within the three target regions-LV cavity, myocardium and background-to a prior learned from a given segmentation of the first frame.

The overlap priors lead to a method which has several advantages over existing ones: (1) shape priors are not needed to obtain satisfying LV tracking because the overlap priors prevent the endo- and epicardium boundaries from spilling, respectively, into the cavity and the background; (2) no assumption is made as to the parametric distributions of intensity/shape data; (3) explicit curve coupling [7, 3] is not required because the proposed functionals and two-step minimization yield an implicit coupling. Those advantages lead to a LV tracking which is more versatile than existing ones because the solution is not bounded to the shape/intensity properties learned from a finite training set.

\section{Formulation}

Let $\mathcal{I}$ be a MR cardiac sequence containing $N$ frames $1, I^{n}: \Omega \subset \mathbb{R}^{2} \rightarrow \mathbb{R}^{+}$, $n \in[1 . . N]$. The purpose of this study is to automatically detect the endocardium (yellow contour in Fig. 1,a) and the epicardium (green contour in Fig. 1,a) of the

${ }^{1}$ The number of frames $N$ is typically equal to 20 or 25 . 
heart for each $n \in[2 . . N]$. We formulate the problem as the evolution of two closed planar parametric curves, $\boldsymbol{\Gamma}_{\text {in }}^{n}(s), \boldsymbol{\Gamma}_{\text {out }}^{n}(s):[0,1] \rightarrow \Omega$, toward, respectively, the endo- and epicardium. The curve evolution equations are sought following the minimization of two original functionals based on the notion of overlap between the intensity distributions within three target regions: (1) the heart cavity $C^{n}$ corresponding to the interior of curve $\boldsymbol{\Gamma}_{i n}^{n}: C^{n}=\mathbf{R}_{\boldsymbol{\Gamma}_{i n}^{n}}$, (2) the myocardium $M^{n}$ corresponding to the region between $\boldsymbol{\Gamma}_{\text {in }}^{n}$ and $\boldsymbol{\Gamma}_{\text {out }}^{n}: M^{n}=\mathbf{R}_{\boldsymbol{\Gamma}_{\text {in }}^{c}}^{n} \cap \mathbf{R}_{\boldsymbol{\Gamma}_{\text {out }}^{n}}$ and (3) the background $B^{n}$ corresponding to the region outside $\boldsymbol{\Gamma}_{\text {out }}^{n}: B^{n}=\mathbf{R}_{\Gamma_{\text {out }}^{n}}^{c}$.

The proposed functionals: For each $\mathbf{R} \in\left\{C^{n}, M^{n}, B^{n}, n=1 . . N\right\}$, define $P_{\mathbf{R}, I}$ as the nonparametric (kernel-based) estimate of intensity distribution within region $\mathbf{R}$ in frame $I \in\left\{I^{n}, n=1 . . N\right\}$

$$
\forall z \in \mathbb{R}^{+}, P_{\mathbf{R}, I}(z)=\frac{\int_{\mathbf{R}} K(z-I(x)) d x}{a_{\mathbf{R}}}
$$

where $a_{\mathbf{R}}$ is the area of region $\mathbf{R}: a_{\mathbf{R}}=\int_{\mathbf{R}} d \mathbf{x}$. Typical choices of $\mathrm{K}$ are the Dirac function and the Gaussian kernel [12]. Let $\mathcal{B}(f / g)$ be the Bhattacharyya coefficient 2 measuring the amount of overlap between two statistical samples $f$ and $g[12$

$$
\mathcal{B}(f / g)=\sum_{z \in \mathbb{R}^{+}} \sqrt{f(z) g(z)}
$$

We assume that a segmentation of the first frame $I^{1}$, i.e., a partition $\left\{C^{1}, M^{1}, B^{1}\right\}$, is given. Consider

$$
\mathbf{B}_{\text {in }}^{n}=\mathcal{B}\left(P_{C^{n}, I^{n}} / P_{M^{1}, I^{1}}\right) ; \mathbf{B}_{\text {out }}^{n}=\mathcal{B}\left(P_{M^{n}, I^{n}} / P_{B^{1}, I^{1}}\right) \quad \forall n \in[1 . . N]
$$

$\mathbf{B}_{i n}^{n}$ measures the overlap between the intensity distributions within the LV cavity and the myocardium in $I^{n}$. $\mathbf{B}_{\text {out }}^{n}$ measures the overlap between the intensity distributions within the myocardium and the background in $I^{n}$. As we will demonstrate experimentally in section [3, $\mathbf{B}_{i n}^{n}$ and $\mathbf{B}_{\text {out }}^{n}$ are approximately constant over a cardiac sequence. Consequently, measures $\mathbf{B}_{\text {in }}^{1}$ and $\mathbf{B}_{\text {out }}^{1}$ estimated from a given segmentation of the first frame in sequence $\mathcal{I}$ can be used as overlap priors to constrain the tracking in frames $I^{2} . . I^{N}$. We adopt a two-step curve evolution for each $n \in[2 . . N]$. First, we evolve the endocardium boundary, $\Gamma_{i n}^{n}$, following the minimization of

$$
\mathcal{F}_{i n}^{n}=\underbrace{\alpha\left(\mathbf{B}_{i n}^{n}-\mathbf{B}_{i n}^{1}\right)^{2}}_{\text {Overlap cavity/myocardium }}+\underbrace{\beta\left(\mu_{i n}^{n}-\mu_{i n}^{1}\right)^{2}}_{\text {Cavity mean }}+\underbrace{\lambda \oint_{\Gamma_{i n}^{n}}\left(g_{n}+c\right) d s}_{\text {Endocardium boundary }}
$$

where $\mu_{i n}^{n}$ is the estimate of intensity mean within $C_{i n}^{n}$ for $n \in[1 . . N]: \mu_{i n}^{n}=$ $\frac{\int_{C_{n}} I^{n} d \mathbf{x}}{a_{C} n}, g_{n}=\frac{1}{1+\left\|\nabla I^{n}\right\|^{2}}$ is an edge indicator function which biases the curve toward high gradient of intensity and $c$ is a constant to enforce curve smoothness.

${ }^{2}$ Note that the values of $\mathcal{B}$ are always in $[0,1]$, where 0 indicates that there is no overlap, and 1 indicates a perfect match. 
$\alpha, \beta$ and $\lambda$ are positive real constants to balance the contribution of each term. Second, we fix the obtained $\boldsymbol{\Gamma}_{i n}^{n}$ and minimize the following functional with respect to the epicardium boundary, i.e., $\boldsymbol{\Gamma}_{\text {out }}$

$$
\mathcal{F}_{\text {out }}^{n}=\underbrace{\alpha\left(\mathbf{B}_{\text {out }}^{n}-\mathbf{B}_{\text {out }}^{1}\right)^{2}}_{\text {Overlap myocardium/background }}+\underbrace{\beta\left(\mu_{\text {out }}^{n}-\mu_{\text {out }}^{1}\right)^{2}}_{\text {Myocardium mean }}+\underbrace{\lambda \oint_{\Gamma_{\text {out }}^{n}\left(g_{n}+c\right) d s}}_{\text {Epicardium boundary }}
$$

where $\mu_{\text {out }}^{n}$ is the estimate of intensity mean within $M_{\text {in }}^{n}$ for $n \in[1 . . N]: \mu_{\text {out }}^{n}=$ $\frac{\int_{M^{n} I^{n} d \mathbf{x}}}{a_{M^{n}}}$. In section 3 we will validate experimentally the usefulness of the proposed overlap terms: for ten different datasets, we will confirm with manual segmentations that $\left(\mathbf{B}_{\text {in }}^{n}-\mathbf{B}_{\text {in }}^{1}\right)^{2}$ and $\left(\mathbf{B}_{\text {out }}^{n}-\mathbf{B}_{\text {out }}^{1}\right)^{2}$ are approximately equal to zero (refer to table 2).

Curve evolution minimization equations: Curve evolutions are obtained by the Euler-Lagrange descent equations. We embed each curve $\boldsymbol{\Gamma} \in\left\{\boldsymbol{\Gamma}_{\text {in }}^{n}, \boldsymbol{\Gamma}_{\text {out }}^{n}\right\}$ in a one-parameter family of curves: $\boldsymbol{\Gamma}(s, t):[0,1] \times \mathbf{R}^{+} \rightarrow \Omega$, and solve the partial differential equations: $\frac{\partial \Gamma_{i n}^{n}(s, t)}{\partial t}=-\frac{\partial \mathcal{F}_{i n}}{\partial \Gamma_{i n}^{n}}, \frac{\partial \Gamma_{\text {out }}^{n}(s, t)}{\partial t}=-\frac{\partial \mathcal{F}_{\text {out }}}{\partial \Gamma_{\text {out }}^{n}}$. After some algebraic manipulations, we obtain the final curve evolution equations

$$
\begin{aligned}
\frac{\partial \boldsymbol{\Gamma}_{\text {in }}^{n}}{\partial t} & =\left\{\frac{\alpha\left(\mathbf{B}_{\text {in }}^{n}-\mathbf{B}_{\text {in }}^{1}\right)}{a_{C^{n}}}\left(\mathbf{B}_{\text {in }}^{n}-\sqrt{\frac{P_{M^{1}, I^{1}}}{P_{C^{n}, I^{n}}}}\right)+\frac{2 \beta\left(\mu_{i n}^{n}-\mu_{\text {in }}^{1}\right)}{a_{C^{n}}}\left(\mu_{\text {in }}^{n}-I^{n}\right)\right. \\
& \left.+\lambda\left[\nabla g_{n} \cdot \boldsymbol{n}_{\text {in }}^{n}-\left(g_{n}+c\right) \kappa_{\text {in }}^{n}\right]\right\} \boldsymbol{n}_{\text {in }}^{n} \\
\frac{\partial \boldsymbol{\Gamma}_{\text {out }}^{n}}{\partial t} & =\left\{\frac{\left(\mathbf{B}_{\text {out }}^{n}-\mathbf{B}_{\text {out }}^{1}\right)}{a_{M^{n}}}\left(\mathbf{B}_{\text {out }}^{n}-\sqrt{\frac{P_{B^{1}, I^{1}}}{P_{M^{n}, I^{n}}}}\right)+\frac{2 \beta\left(\mu_{\text {out }}^{n}-\mu_{\text {out }}^{1}\right)}{a_{M^{n}}}\left(\mu_{\text {out }}^{n}-I^{n}\right)\right. \\
& \left.+\lambda\left[\nabla g_{n} \cdot \boldsymbol{n}_{\text {out }}^{n}-\left(g_{n}+c\right) \kappa_{\text {out }}^{n}\right]\right\} \boldsymbol{n}_{\text {out }}^{n}
\end{aligned}
$$

where $\boldsymbol{n}_{i n}^{n}$ and $\boldsymbol{n}_{\text {out }}^{n}$ are the outward unit normals to, respectively, $\boldsymbol{\Gamma}_{\text {in }}^{n}$ and $\boldsymbol{\Gamma}_{\text {out }}^{n}$. $\kappa_{\text {in }}^{n}$ and $\kappa_{\text {out }}^{n}$ are the mean curvature functions of, respectively, $\boldsymbol{\Gamma}_{\text {in }}^{n}$ and $\boldsymbol{\Gamma}_{\text {out }}^{n}$. Partition $\left(C^{n}, M^{n}, B^{n}\right)$ of frame $I^{n}$ is obtained from $\boldsymbol{\Gamma}_{\text {in }}^{n}$ and $\boldsymbol{\Gamma}_{\text {out }}^{n}$ at convergence i.e., when $t \rightarrow \infty$. The level-set framework [1] is used to implement the evolution equations in (6). The level-set implementation has well-known advantages over explicit curve discretization and can be effected by stable numerical schemes.

\section{Experiments}

In the following, we first give a typical example which demonstrates clearly the advantage of using overlap constraints over the commonly used ML constraints. Then, we describe a statistical performance evaluation of the method by comparisons with manual segmentations and other variational methods [2, [3]. We also evaluate the statistics of the proposed overlap priors/measures over 


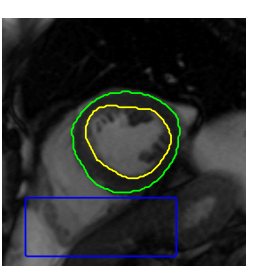

(a)

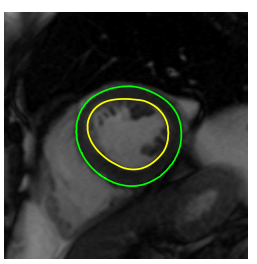

(d)

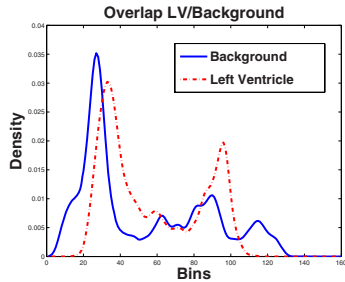

(b)

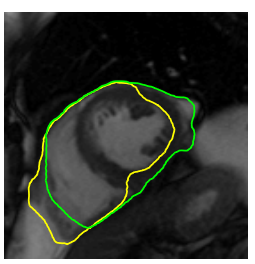

(e)

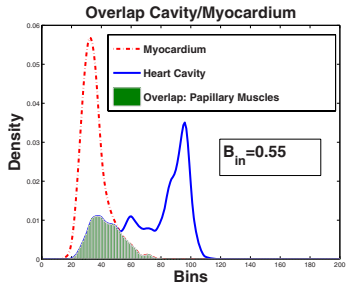

(c)

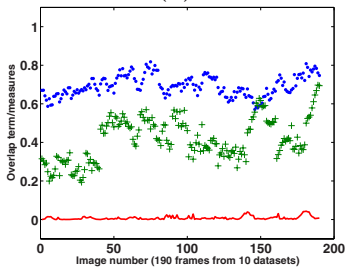

(f)

Fig. 1. Advantage of overlap constraints: (a) manual segmentation by a radiologistyellow curve: endocardium, green curve: epicardium; (b) overlap between the distributions within the LV and the nearby background (region inside the blue curve in a); overlap between the distributions within the cavity and the myocardium; (d) segmentations obtained with and overlap constraint, i.e., with our method; (e) segmentations obtained with a $M L$ constraint. Both constraints were used without shape priors. (f) The proposed overlap priors/measures versus the frame number (190 frames) in 10 manually segmented datasets: $\mathbf{B}_{\text {out }}^{n}$ (blue points); $\mathbf{B}_{i n}^{n}$ (green markers); $\left(\mathbf{B}_{\text {out }}^{n}-\mathbf{B}_{\text {out }}^{1}\right)^{2}+\left(\mathbf{B}_{\text {in }}^{n}-\mathbf{B}_{\text {in }}^{1}\right)^{2}$ (continuous red line).

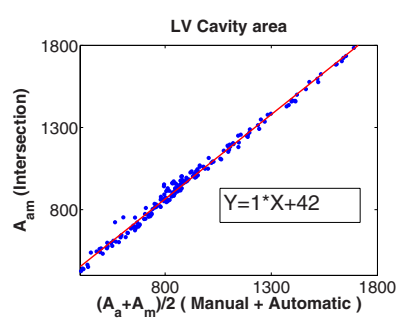

(a)

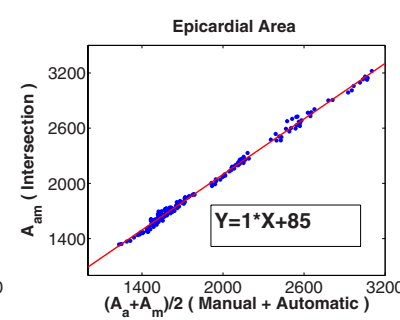

(b)

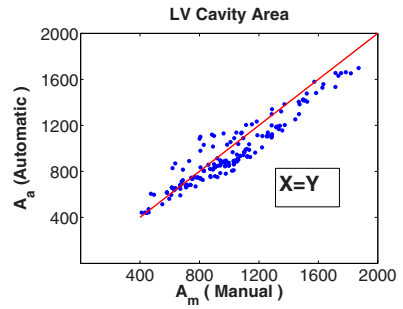

(c)

Fig. 2. Comparisons of manual and automatic segmentations in 190 images from 10 datasets. $\mathbf{A}_{\mathbf{a} m}$ versus $\frac{\mathbf{A}_{\mathbf{a}}+\mathbf{A}_{\mathbf{m}}}{2}$ : (a) cavity area $(D M=\mathbf{0 . 9 2} \pm 0.03)$; (b) epicardial area $(D M=0.94 \pm 0.01)$. (c) Automatic versus manual LV cavity areas.

several datasets. Finally, we give a representative sample of the results for visual inspection.

(1) Overlap constraint vs. ML intensity-driven constraint: Figure 1 (a) shows a typical example of a MR mid-cavity frame. It depicts the expected segmentations of the LV cavity (region inside the yellow curve) and the epicardial region (region inside the green curve). Figs. 1 b and c illustrate the significant 
overlap between the distributions within the three target regions: cavity, myocardium and background. No shape priors were added for a fair comparison between overlap and ML constraints. For both constraints, model distributions of regions were estimated from the same pesegmented frame. With a ML intensitydriven constraint, parts of the background, which have intensity profiles similar to the cavity and the myocardium, are included inside the final curves (refer to Fig. 1.e). The use of geometric constraints, such as shape priors, in conjunction with ML constraints is inevitable to obtain satisfying results [5], 6]. By contrast, using an overlap constraint delineates accurately the cavity and the LV (refer to Fig. 11), thereby removing the need of shape priors.

Table 1. Comparisons of manual and automatic segmentations: Dice metrics and correlation coefficients. The higher the Dice metric, the better the segmentation.

\begin{tabular}{|c|c|c||c|c|c|}
\hline Dice metrics & Mean & Std & Correlation & LV cavity areas & Epicardial areas \\
\hline This method & 0.93 & 0.02 & This method & 0.94 & 0.96 \\
\hline Method in [2] & 0.81 & 0.16 & Method in [3] & 0.89 & 0.87 \\
\hline
\end{tabular}

Table 2. Statistics of the overlap priors/measures (expressed as mean \pm std) over ten datasets. The overlap priors are approximately equal to zero.

\begin{tabular}{|c|c|c|c|}
\hline $\mathbf{B}_{\text {out }}^{n}$ & $\mathbf{B}_{\text {in }}^{n}$ & $\left(\mathbf{B}_{\text {out }}^{n}-\mathbf{B}_{\text {out }}^{1}\right)^{2},(n>1)$ & $\left(\mathbf{B}_{i n}^{n}-\mathbf{B}_{\text {in }}^{1}\right)^{2},(n>1)$ \\
\hline $0.69 \pm 0.05$ & $0.42 \pm 0.17$ & $1.9\left(10^{-3}\right) \pm 2.4\left(10^{-3}\right)$ & $6.5\left(10^{-3}\right) \pm 8.5\left(10^{-3}\right)$ \\
\hline
\end{tabular}

(2) Statistical performance evaluation: The performance of the proposed variational technique was evaluated by comparisons with independent manual segmentations approved by an experienced cardiologist. We applied the method to $2 \mathrm{D}$ mid-cavity $\mathrm{MR}$ sequences obtained from 10 patients, i.e., 10 different datasets: 190 frames were automatically segmented. The free parameters were unchanged for all the datasets: $\alpha=1000, \beta=10, \lambda=0.1, c=10$. Curve initializations and estimation of $B_{i n}^{1}, B_{o u t}^{1}, \mu_{i n}^{1}$, and $\mu_{\text {out }}^{1}$ were obtained from a userprovided segmentation of the first frame in each sequence. Two clinically important measures were evaluated for performance appraisal: LV cavity area and $L V$ epicardial area. Area measurements are expressed as the number of pixels within the region. We first used the Dice Metric (DM) to measure the similarity between manual and automatic segmentations. Let $\mathbf{A}_{\mathbf{a}}, \mathbf{A}_{\mathbf{m}}$ and $\mathbf{A}_{\mathbf{a} m}$ be the areas of, respectively, the automatically detected region, the corresponding hand-labeled region and the intersection between them. $D M$ is given by $\frac{2 \mathbf{A}_{\mathbf{a} m}}{\mathbf{A}_{\mathbf{a}}+\mathbf{A}_{\mathbf{m}}}$ 2] 3 . Our algorithm yielded a $D M$ equal to $\mathbf{0 . 9 3} \pm \mathbf{0 . 0 2}$ for all the data $(D M$ is expressed as mean \pm standard deviation). We obtained $D M$ equal to $\mathbf{0 . 9 2} \pm \mathbf{0 . 0 3}$ for the LV cavity areas and $D M$ equal to $\mathbf{0 . 9 4} \pm \mathbf{0 . 0 1}$ for the epicardial areas. Linear regression was also used to assess the differences between $\mathbf{A}_{\mathbf{a} m}$ and $\frac{\mathbf{A}_{\mathbf{a}}+\mathbf{A}_{\mathbf{m}}}{2}$

${ }^{3} D M$ is always in $[0,1] . D M$ equal to 1 indicates a perfect match between manual and automatic segmentation. 


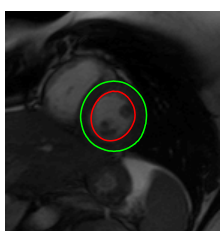

$s 1 f 5$

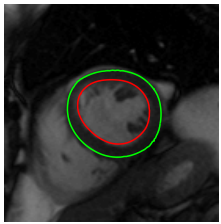

$s 2 f 3$

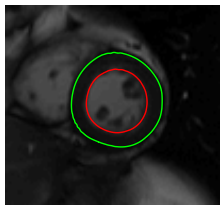

$s 3 f 5$

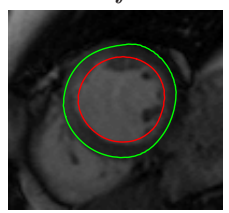

$s 4 f 5$

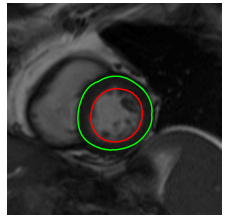

$s 5 f 3$

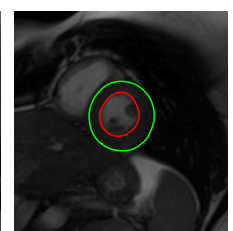

$s 1 f 7$

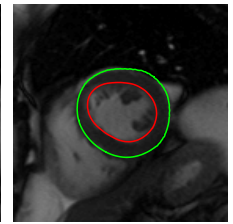

$s 2 f 5$

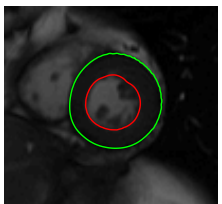

$s 3 f 7$

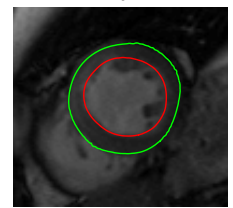

$s 4 f 7$

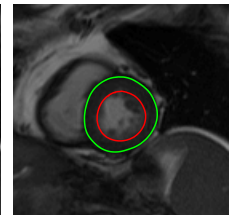

$s 5 f 5$

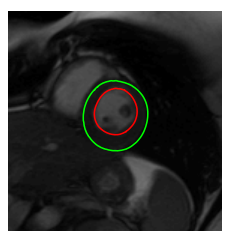

$s 1 f 9$

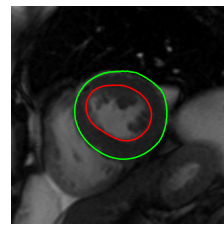

$s 2 f 7$

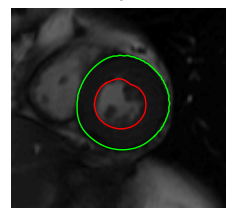

$s 3 f 9$

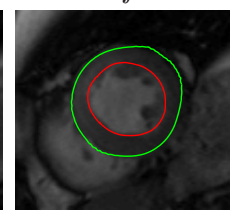

$s 4 f 9$

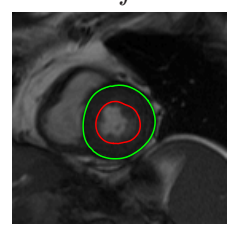

$s 5 f 9$

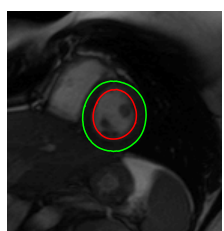

$s 1 f 11$

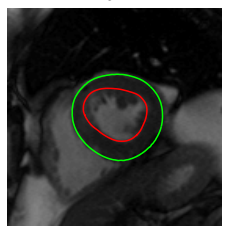

$s 2 f 13$

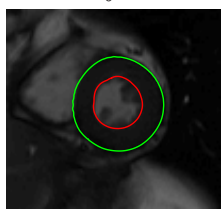

s $3 f 12$

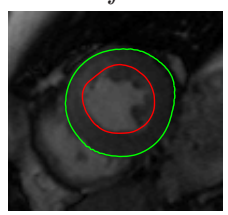

$s 4 f 12$

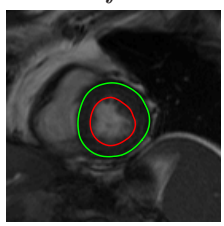

$s 5 f 11$

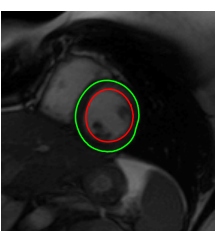

$s 1 f 13$

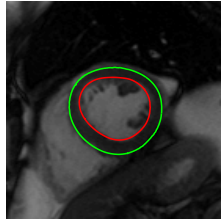

$s 2 f 17$

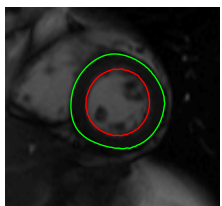

$s 3 f 15$

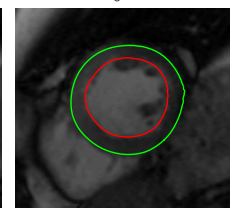

$s 4 f 15$

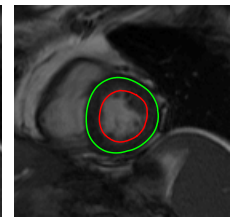

$s 5 f 13$

Fig. 3. Results for five MR sequences. Each row depicts the results for one sequence. $\alpha=1000, \beta=10, \lambda=0.1, c=10 . \boldsymbol{\Gamma}_{\text {in }}^{n}$ : red curve, $\boldsymbol{\Gamma}_{\text {out }}^{n}$ : green curve.

(refer to Figs. 2 a and b). The smaller these differences, the higher the similarities between manual and automatic segmentations. The LV cavity and epicardial regression lines did not differ significantly from the identity line. We also report good correlation coefficients between manual and automatic endo- and epicardial areas (refer to table 1). To assess the differences between manual and automatic LV cavity areas, we give the linear regression plot in Fig. 2 c, displayed with the identity line.

To bear comparisons with other recent variational approaches to LV tracking, we give in table 1 the Dice metrics and correlation coefficients reported, respectively, in [2] and 3. Our method leads to a significant improvement in 
accuracy over the variational level set methods in [2, 3]. Although the proposed functional does not embed shape knowledge, it compares very well with existing methods [2]-7] which, in most cases, use shape priors. Different from ML intensity-driven constraints, the proposed overlap constraints measure the similarities between the intensity distributions within the cardiac regions, thereby removing the need of shape constraints.

In Fig. 1,f, we plotted the proposed overlap priors/measures versus the frame number (190 frames) using manual segmentations from 10 datasets. As reported in table 2, the overlap priors in Eqs. (4) and (5) are approximately equal to zero (refer to the continuous line in Fig. 1.f). This validates the usefulness of such priors for the LV tracking. It is also interesting to notice that overlap measures $B_{i n}^{n}$ and $B_{\text {out }}^{n}$ do not vary much over different patients (refer to Fig. 11. f).

In Fig. 3, we give a representative sample of the results with five sequences $(s 1-s 5)$. sxfy depicts the tracking obtained for frame $y$ in sequence $x$. The red and green curves represent, respectively, $\boldsymbol{\Gamma}_{\text {in }}^{n}$ and $\boldsymbol{\Gamma}_{\text {out }}^{n}$ at convergence.

\section{Conclusion}

We investigated overlap priors for variational LV tracking in MR sequences. Quantitative evaluation showed the advantages of overlap priors over existing intensity-driven constraints. Although neither shape priors nor curve coupling were used, the results correlated well with manual segmentations and the method compared favorably with other methods.

\section{References}

1. Cremers, D., Rousson, M., Deriche, R.: A Review of Statistical Approaches to Level Set Segmentation: Integrating Color, Texture, Motion and Shape. International Journal of Computer Vision 62, 249-265 (2007)

2. Lynch, M., Ghita, O., Whelan, P.F.: Segmentation of the Left Ventricle of the Heart in 3-D+t MRI Data Using an Optimized Nonrigid Temporal Model. IEEE Transactions on Medical Imaging 27(2), 195-203 (2008)

3. Lynch, M., Ghita, O., Whelan, P.F.: Left-ventricle myocardium segmentation using a coupled level-set with a priori knowledge. Computerized Medical Imaging and Graphics 30, 255-262 (2006)

4. Jolly, M.-P.: Automatic Segmentation of the Left Ventricle in Cardiac MR and CT Images. International Journal of Computer Vision 70(2), 151-163 (2006)

5. Kohlberger, T., Cremers, D., Rousson, M., Ramaraj, R., Funka-Lea, G.: 4D Shape Priors for a Level Set Segmentation of the Left Myocardium in SPECT Sequences. In: Larsen, R., Nielsen, M., Sporring, J. (eds.) MICCAI 2006. LNCS, vol. 4190, pp. 92-100. Springer, Heidelberg (2006)

6. Sun, W., Çetin, M., Chan, R., Reddy, V., Holmvang, G., Chandar, V., Willsky, A.: Segmenting and Tracking the Left Ventricle by Learning the Dynamics in Cardiac Images. Information Processing in Medical Imaging, 553-565 (2005)

7. Paragios, N.: A Variational Approach for the Segmentation of the Left Ventricle in Cardiac Image Analysis. IJCV 50(3), 345-362 (2002) 
8. Cootes, T., Edwards, G., Taylor, C.: Active Appearance Models. IEEE Transactions on Pattern Analysis and Machine Intelligence 23(6), 681-685 (2001)

9. Andreopoulos, A., Tsotsos, J.K.: Efficient and Generalizable Statistical Models of Shape and Appearance for Analysis of Cardiac MRI. Medical Image Analysis (in press)

10. Zambal, S., Hladůvka, J., Bühler, K.: Improving Segmentation of the Left Ventricle Using a Two-Component Statistical Model. In: Larsen, R., Nielsen, M., Sporring, J. (eds.) MICCAI 2006. LNCS, vol. 4190, pp. 151-158. Springer, Heidelberg (2006)

11. Mitchell, S.C., Lelieveldt, B.P.F., van der Geest, R.J., Bosch, H.G., Reiber, J.H.C., Sonka, M.: Multistage Hybrid Active Appearance Model Matching: Segmentation of Left and Right Ventricles in Cardiac MR Images. IEEE Transactions on Medical Imaging 20(5), 415-423 (2001)

12. Michailovich, O.V., Rathi, Y., Tannenbaum, A.: Image Segmentation Using Active Contours Driven by the Bhattacharyya Gradient Flow. IEEE Transactions on Image Processing 16(11), 2787-2801 (2007)

13. Zhang, T., Freedman, D.: Improving performance of distribution tracking through background mismatch. IEEE Transactions on Pattern Analysis and Machine Intelligence 27(2), 282-287 (2005)

14. Ben Ayed, I., Li, S., Ross, I.: Tracking Distributions with an Overlap Prior. In: IEEE Computer Society Conference on Computer Vision and Pattern Recognition (CVPR 2008), Anchorage, Alaska (2008)

15. Freedman, D., Radke, R.J., Zhang, T., Jeong, Y., Lovelock, D.M., Chen, G.T.Y.: Model-Based Segmentation of Medical Imagery by Matching Distributions. IEEE Transactions on Medical Imaging 24(3), 281-292 (2005) 